\title{
Recent developments on HYDJET/PYQUEN event generators and novel jet quenching observables at LHC
}

\section{I.P. Lokhtin*, L.V. Malinina, S.V. Petrushanko, A.M. Snigirev}

D.V. Skobeltsyn Institute of Nuclear Physics, M.V. Lomonosov Moscow State University, Moscow, Russia

E-mail: Igor@Lokhtin@cern.ch

\section{Arsene, $\mathrm{K}$. Tywoniuk}

The Department of Physics, University of Oslo, Norway

\begin{abstract}
The recent developments on PYQUEN, HYDJET and HYDJET++ event generators are presented. The partonic energy loss model PYQUEN is implemented as the modification of the "standard" jet event obtained with the generator of hadron-hadron interactions PYTHIA. HYDJET and HYDJET++ are the Monte-Carlo event generators for the simulation of relativistic heavy ion AA collisions considered as a superposition of the soft, hydro-type state and the hard, multi-parton fragmentation. HYDJET++ model is the development and continuation of HYDJET generator, and it includes more detailed treatment of the "thermal" hadronic state generated on the chemical and thermal freeze-out hypersurfaces (represented by the parameterization of relativistic hydrodynamics with preset freeze-out conditions), collective flow effects and decays of hadronic resonances. Some applications of above models to novel jet quenching observables are discussed.
\end{abstract}

High-pT Physics at LHC - Tokaj'08

March 16 - 192008

Tokaj, Hungary

\footnotetext{
* Speaker.

${ }^{\dagger}$ On leave from the Institute for Space Sciences, Bucharest, Romania

$\doteqdot$ Curren affiliation: Departamento de Física de Partículas, Universidad de Santiago de Compostela, Santiago de Compostela, Spain
} 


\section{Introduction}

The experimental and phenomenological study of multi-particle production in relativistic heavy ion collisions is expected to provide valuable information on the dynamical behaviour of stronglyinteracting matter in the form of a quark-gluon plasma (QGP), as predicted by lattice Quantum Chromodynamics (QCD) calculations. Ongoing and future experimental studies in a wide range of heavy ion beam energies require the development of new Monte-Carlo (MC) event generators and improvement of existing ones. Especially for experiments at the CERN Large Hadron Collider (LHC), because of very high parton and hadron multiplicities, one needs fast (but realistic) MC tools for heavy ion event simulations [1, 2, 3, 4]. The main advantage of MC technique for the simulation of high-multiplicity hadroproduction is that it allows a visual comparison of theory and data, including if necessary the detailed detector acceptances, resolutions and responses. The realistic MC event generator has to include maximum possible number of observed physical effects, which are important for the determination of event topologies: from bulk properties of soft hadroproduction (domain of low transverse momenta $p_{T} \lesssim 1 \mathrm{GeV} / c$ ), such as collective flows, to hard multi-parton production in hot and dense QCD-matter, which reveals itself in spectra of high- $p_{T}$ particles and hadronic jets. Moreover, the role of hard and semi-hard particle production at LHC can be significant even for bulk properties of created matter, and hard probes of QGP became clearly observable in various new channels [4]. In most of the available MC heavy ion event generators, the simultaneous treatment of collective flow effects for soft hadroproduction and hard multi-parton in-medium production (medium-induced partonic rescattering and energy loss, so called 'jet quenching") is lacking. Thus, in order to analyze existing data on low and high-p ${ }_{T}$ hadron production, to test the sensitivity of physical observables at the upcoming LHC experiments (and other future heavy ion facilities) to the QGP formation, and study the experimental capabilities of constructed detectors, the development of adequate and fast MC models for simultaneous collective flow and jet quenching simulations is necessary. HYDJET and HYDJET++ event generators includes detailed treatment of hard multi-parton production with taking into account known medium effects (such as jet quenching and nuclear shadowing), as well as soft hadroproduction.

\section{PYQUEN event generator}

The event generator for medium-modified nucleon-nucleon collisions PYQUEN [5] is the modification of the "standard" jet event obtained with the generator of hadron-hadron interactions PYTHIA [6]. The details of PYQUEN partonic energy loss model can be found in [7]. The event-by-event simulation procedure in PYQUEN includes the following steps.

1. Generation of initial partonic state production by calling PYTHIA (parton fragmentation being switched off). The spatial vertex of a jet production $\mathrm{J}(x=r \cos \psi, y=r \sin \psi)$ is generated according to the distribution

$$
\frac{d N^{\mathrm{jet}}}{d \psi r d r}(b)=\frac{T_{A}\left(r_{1}\right) \cdot T_{A}\left(r_{2}\right)}{T_{A A}(b)}
$$

where $b$ is the impact parameter of a heavy ion collision, $T_{A}$ and $T_{A A}$ are the standard nuclear thickness and nuclear overlap functions, $r_{1,2}(b, r, \psi)$ are the distances between the nucleus centers and the vertex $\mathrm{J}$, and $r$ is the distance from the nuclear collision axis to vertex $\mathrm{J}$. 
2. Then initial PYTHIA-produced partonic state is modified by medium-induced radiative and collisional energy loss. For each hard parton the following rescattering scheme is applied in the course of a proper time $\tau$.

- Calculation of the scattering cross section $\sigma\left(\tau_{i}\right)=\int d t d \sigma / d t$ and generation of the transverse momentum transfer $t\left(\tau_{i}\right)$ in the $i$-th scattering in the high-momentum trasfer limit.

- Generation of the displacement between the $i$-th and $(i+1)$-th scatterings, $l_{i}=\left(\tau_{i+1}-\tau_{i}\right)$ :

$$
\frac{d P}{d l_{i}}=\lambda^{-1}\left(\tau_{i+1}\right) \exp \left(-\int_{0}^{l_{i}} \lambda^{-1}\left(\tau_{i}+s\right) d s\right), \lambda^{-1}(\tau)=\sigma(\tau) \rho(\tau),
$$

and calculation of the corresponding transverse distance, $l_{i} p_{T} / E$. Here $\lambda=1 /(\sigma \rho)$ is inmedium mean free path, $\rho \propto T^{3}$ is the medium density at the temperature $T, \sigma$ is the integral cross section for parton interaction in the medium.

- Generation of the energy of a radiated in the $i$-th scattering gluon, $\omega_{i}=\Delta E_{\mathrm{rad}, i}$, according to BDMS radiation spectrum $[8,9]$ and its emission angle $\theta_{i}$ relative to the parent parton determined according to the selected by user parameterization.

- Calculation of the collisional energy loss in the $i$-th scattering

$$
\Delta E_{\mathrm{col}, i}=\frac{t_{i}}{2 m_{0}}
$$

where energy of "thermal" medium parton $m_{0}$ is generated according to the isotropic Boltzmann distribution at the temperature $T\left(\tau_{i}\right)$.

- Reducing the parton energy by collisional and radiative loss per each $i$-th scattering,

$$
\Delta E_{\mathrm{tot}, i}=\Delta E_{\mathrm{col}, i}+\Delta E_{\mathrm{rad}, i}
$$

and changing the parton momentum direction by adding the transverse momentum kick due to elastic scattering $i$,

$$
\Delta k_{t, i}^{2}=\left(E-\frac{t_{i}}{2 m_{0 i}}\right)^{2}-\left(p-\frac{E}{p} \frac{t_{i}}{2 m_{0 i}}-\frac{t_{i}}{2 p}\right)^{2}-m_{p}^{2} .
$$

- Going to the next rescattering, or halting the rescattering if one of the following two conditions is fulfilled: (a) the parton escapes the hot QGP zone, i.e. the temperature in the next point $T\left(\tau_{i+1}, r_{i+1}, \eta_{i+1}\right)$ becomes less than $T_{c}=200 \mathrm{MeV}$; or $(b)$ the parton loses so much energy that its transverse momentum $p_{T}\left(\tau_{i+1}\right)$ drops below the average transverse momentum of the "thermal" constituents of the medium, $2 T\left(\tau_{i+1}\right)$. In latter case, such a parton is considered to be "thermalized" and its momentum in the rest frame of the quark-gluon fluid is generated from the random "thermal" distribution, $d N / d^{3} p \propto \exp (-E / T)$, boosted to the center-of-mass of the nucleus-nucleus collision.

3. At the end of each NN collision, adding new (in-medium emitted) gluons to the PYTHIA parton list and rearrangement of partons to update string formation with the subroutine PYJOIN are performed. An additional gluon is included in the same string as its "parent", and colour connections of such gluons are re-ordered by their $z$-coordinates along the string. Then final hadrons are formed by calling standard PYTHIA fragmentation routine PYEXEC. 


\section{HYDJET and HYDJET++ event generators}

HYDJET [7, 10] and HYDJET++ $[11,12]$ are the Monte-Carlo event generators for the simulation of relativistic heavy ion AA collisions considered as a independent superposition of the soft, hydro-type component and the hard, multi-parton fragmentation. The hard parts of HYDJET++ and HYDJET are identical, but soft parts are different. The treatment of soft component in HYDJET is rather oversimplified. It considers pions, kaons and protons/neutrons only, the hadron spectrum being generated as the convolution of thermal motion and collective flow [7]. The soft part of HYDJET++ is the "thermal" hadronic state generated on the chemical and thermal freezeout hypersurfaces represented by the parameterization of relativistic hydrodynamics with preset freeze-out conditions(the adapted C++ code FAST MC [13, 14]). It includes longitudinal, radial and elliptic flow effects and decays of hadronic resonances. The HYDJET was written in Fortran. The main program of HYDJET++ was written in C++ under the ROOT environment [15] (but there is the Fortran-written hard part included in the generator structure as a separate directory).

Before any event generation, HYDJET/HYDJET++ run starts from PYTHIA initialization at given c.m.s. energy per nucleon pair $\sqrt{s}$, and then it calculates the total inelastic $\sigma_{N N}^{\text {in }}(\sqrt{s})$ and hard scattering $\sigma_{N N}^{\text {hard }}\left(\sqrt{s}, p_{T}^{\min }\right) \mathrm{NN}$ cross sections with the minimum transverse momentum transfer $p_{T}^{\min }$. If the impact parameter $b$ of heavy ion AA collision is not fixed by user, its value $b$ is generated in each event between minimum and maximum values in accordance with the differential inelastic AA cross section:

$$
\frac{d^{2} \sigma_{\text {in }}^{A A}}{d^{2} b}(b, \sqrt{s})=\left[1-\left(1-\frac{1}{A^{2}} T_{A A}(b) \sigma_{N N}^{\mathrm{in}}(\sqrt{s})\right)^{A^{2}}\right] .
$$

If the impact parameter $b$ is fixed, then its value in each event is equal to the corresponding input parameter. After specification of $b$ for each given event, mean numbers of binary NN sub-collisions $\overline{N_{\text {bin }}}$ and nucleons-participants $\overline{N_{\text {part }}}$ are calculated:

$$
\begin{aligned}
& \overline{N_{\text {bin }}}(b, \sqrt{s})=T_{A A}(b) \sigma_{N N}^{\text {in }}(\sqrt{s}), \\
& \overline{N_{\text {part }}}(b, \sqrt{s})=\int_{0}^{2 \pi} d \psi \int_{0}^{\infty} r d r T_{A}\left(r_{1}\right)\left[1-\exp \left\{\sigma_{N N}^{\text {in }}(\sqrt{s}) T_{A}\left(r_{2}\right)\right\}\right] .
\end{aligned}
$$

The next step is the simulation of properly particle production in the event. The soft, hydro-type state and hard, multi-parton state are simulated independently. When the generation of soft and hard components in each event at given $b$ is completed, the event record (information about coordinates and momenta of primordial particles, decay products of unstable particles and stable particles) is formed as the junction of these two independent event outputs.

\subsection{Generation of hard multi-parton state}

1. Calculation of the number of $\mathrm{NN}$ sub-collisions $N_{A A}^{\text {jet }}$ producing hard parton-parton scatterings of selected type with $p_{T}>p_{T}^{\min }$, according to the binomial distribution around its mean value (without nuclear shadowing correction yet, $S=1$ ). For this purpose, each from $N_{\text {bin }}$ sub-collisions is treated by the comparison of random number $\xi_{i}$ generated uniformly in the interval $[0,1]$ with the probability $P_{\text {hard }}=\sigma_{N N}^{\text {hard }} / \sigma_{N N}^{\text {in }}$ produce the hard process. The $i$-th sub-collision is accepted if $\xi_{i}<P_{\text {hard }}$, and is rejected in the opposite case. 
2. Selecting the type of hard NN sub-collision ( $\mathrm{pp}$, np or $\mathrm{nn}$ ) in accordance with the phenomenological formula for number of protons $Z$ in the stable nucleus $A, Z=A /\left(1.98+0.015 A^{2 / 3}\right)$. For this purpose, every from $N_{\text {jet }}$ "successful" sub-collisions is treated by the comparison of two random numbers $\xi_{i}^{1}$ and $\xi_{i}^{2}$ generated uniformly in the interval $[0,1]$ with the probability $Z / A$. The proton-proton sub-collision is selected if $\xi_{i}{ }^{1}, \xi_{i}^{2}<Z / A$, neutron-neutron sub-collision - if $\xi_{i}{ }^{1}, \xi_{i}{ }^{2}>Z / A$, and proton-neutron sub-collision — in other cases.

3. Generation of multi-parton production in $N_{\text {jet }}$ hard NN sub-collisions by calling PYQUEN $N_{\text {jet }}$ times (see the previous section).

4. If nuclear shadowing is switched on and beam ions are $\mathrm{Pb}, \mathrm{Au}, \mathrm{Pd}$ or $\mathrm{Ca}$, each hard $\mathrm{NN}$ sub-collision is treated by the comparison of random number $\xi_{i}$ generated uniformly in the interval $[0,1]$ with the shadowing factor $S$ taken from the available parameterization [16]. It is determined by the type of initially scattered hard partons, momentum fractions taken by the partons at the initial hard interaction $x_{1,2}$, the square of transverse momentum transfer in the hard scattering $Q^{2}$, and the transverse position of jet production vertex relative to the the nucleus centers $r_{1,2}$. The given sub-collision is accepted if $\xi_{i}<S$, and is rejected in the opposite case.

5. Formation of hadrons for each "accepted" hard NN sub-collision with PYTHIA (parton fragmentation being switched on), and final junction of $N_{\text {jet }}$ sub-events to common array using standard PYTHIA event output format.

\subsection{Generation of soft "thermal" state}

The details of physics models used to generate thermal hadronic states in HYDJET and HYDJET++ can be found in [7] and in [11] respectively. The following MC simulation procedure is applied to generate the "soft" thermal component in HYDJET++ (in HYDJET only step (4) for pions, kaons and protons/neutrons is used).

1. Initialization of the chemical freeze-out parameters. It includes the calculation of particle number densities. So far, only the stable hadrons and resonances consisting of $u, d, s$ quarks are taken into account from the SHARE particle data table [17].

2. Initialization of the thermal freeze-out parameters (if $T^{\text {th }}<T^{\mathrm{ch}}$ ). It includes the calculation of chemical potentials and particle number densities.

3. Calculation of the effective volume of hadron emission region $V_{\text {eff }}(b)$, the fireball transverse radius $R_{f}(b)$, the freeze-out proper time $\tau_{f}(b)$ and emission duration $\Delta \tau_{f}(b)$, and the mean multiplicity of each particle species. Then the multiplicity is generated around its mean value according to the Poisson distribution.

4. For each hadron the following procedure to generate its four-momentum is applied.

- Generation of four-coordinates of a hadron in the fireball rest frame

$x^{\mu}=\{\tau \cosh \eta, r \cos \phi, r \sin \phi, \tau \sinh \eta\}$ on each freeze-out hypersurface segment $\tau(r)$ for 
the element $d^{3} \sigma_{\mu} u^{\mu}=d^{3} \sigma_{0}^{*}=n_{0}^{*}(r)\left|1-(d \tau / d r)^{2}\right|^{1 / 2} \tau(r) d^{2} r d \eta$, assuming $n_{0}^{*}$ and $\tau$ functions of $\mathrm{r}$ (i.e., independent of $\eta, \phi$ ). It includes sampling uniformly distributed $\phi$ in the interval $[0,2 \pi]$, generating $\eta$ according to the Gaussian distribution $\exp \left(-\eta^{2} / 2 \eta_{\max }^{2}\right)$ and $r$ in the interval $\left.\left[0, R_{f}(b)\right]\right)$ using a $100 \%$ efficient procedure similar to the ROOT routine GetRandom().

- Calculation of the corresponding collective flow four-velocities.

- Generation of the three-momentum of a hadron in the fluid element rest frame $p^{*}\left\{\sin \theta_{p}^{*} \cos \phi_{p}^{*}, \sin \theta_{p}^{*} \sin \phi_{p}^{*}, \cos \theta_{p}^{*}\right\}$ according to the equilibrium distribution function $f_{i}^{\mathrm{eq}}\left(p^{0 *} ; T, \mu_{i}\right) p^{* 2} d p^{*} d \cos \theta_{p}^{*} d \phi_{p}^{*}$ by sampling uniformly distributed $\cos \theta_{p}^{*}$ in the interval $[-1,1]$ and $\phi_{p}^{*}$ in the interval $[0,2 \pi]$, and generating $p^{*}$ using a $100 \%$ efficient procedure (similar to ROOT routine GetRandom()).

- The standard von Neumann rejection/acceptance procedure to account the difference between the true probability $W_{\sigma, i}^{*} d^{3} \sigma d^{3} \vec{p}^{*} / p^{0 *}$ and the probability $n^{0 *} f_{i}^{\mathrm{eq}}\left(p^{0 *} ; T, \mu_{i}\right) d^{2} \vec{r} d \eta d^{3} \vec{p}^{*}$ corresponding to the previous simulation steps. For this purpose, the residual weight is calculated [13]:

$$
W_{i}^{\mathrm{res}}=\frac{W_{\sigma, i}^{*} d^{3} \sigma}{n^{0 *} p^{0 *} f_{i}^{\mathrm{eq}} d^{2} \vec{r} d \eta}=\tau\left(1-\frac{\overrightarrow{n^{*}} \vec{p}^{*}}{n^{0 *} p^{0 *}}\right) .
$$

Then the simulated hadron four-coordinate and four-momentum is treated by the comparison of $W_{i}^{\text {res }}$ with the random number $\xi_{i}$ generated uniformly in the interval $\left[0, \max \left(W_{i}^{\text {res }}\right)\right]$. The $i$-th hadron is accepted if $\xi_{i}<W_{i}^{\text {res }}$, and is rejected in the opposite case (then the generation of its four-coordinate and four-momentum is tried again).

- Boost of the hadron four-momentum in the center mass frame of the event using the velocity field $\vec{v}(x)$, that is,

$$
p^{0}=\gamma\left(p^{0 *}+\vec{v}^{*}\right), \quad \vec{p}=\vec{p}^{*}+\gamma(1+\gamma)^{-1}\left(p^{0 *}+p^{0}\right) \vec{v} .
$$

Note that a high generation speed for this algorithm is achieved because of almost $100 \%$ generation efficiency due to nearly uniform residual weights $W_{i}^{\text {res }}$.

5. Formation of final hadrons from the two- and three-body decays of resonances with the random choice of decay channels according to the branching ratios taken from SHARE particle data files. The two- and three- particle "decayer" programs were developed by N.S. Amelin for FAST MC generator [13, 14], and have been implemented in HYDJET++.

\section{Physics applications of PYQUEN and HYDJET/HYDJET++}

It was demonstrated in [7] that PYQUEN and HYDJET models are capable of reproducing the main features of jet quenching pattern in Au+Au collisions at RHIC (high- $p_{T}$ hadron spectra and the suppression of azimuthal back-to-back correlations). HYDJET++ describes well even wider range of various hadronic observables measured in heavy ion collisions at RHIC for different centrality sets and kinematic ranges: ratio of hadron yields, pseudorapidity and transverse momentum spectra (for both low- and high- $p_{T}$ domains), elliptic flow coefficients $v_{2}\left(p_{T}\right)$, femtoscopic correlations [11]. Thus the above generators can be applied for the various simulation studies at LHC energies. The set of PYQUEN/HYDJET applications to novel jet quenching observables in heavy 
ion collisions at the LHC was summarized in [4]. Let us enumerate the main predicted observables (all numbers were obtained for central $\mathrm{Pb}+\mathrm{Pb}$ collisions at $\sqrt{s}=5.5 \mathrm{ATeV}$ and hadronic jets of a cone size $R=0.5$ and transverse energy $E_{T}>100 \mathrm{GeV}$ ).

Nuclear modification factor for jets. The jet nuclear modification factor is defined as a ratio of jet yields in $A A$ and pp collisions normalized on the number of binary nucleon-nucleon collisions. The predicted by PYQUEN/HYDJET jet suppression factor (due to partial gluon bremsstrahlung out of jet cone and collisional loss) is about 2 and almost independent on jet energy. It is clear that the measured jet nuclear modification factor will be very sensitive to the fraction of partonic energy loss carried out of the jet cone.

Medium-modified jet fragmentation function. The "jet fragmentation function" (JFF), $D(z)$, is defined as the probability for a given product of the jet fragmentation to carry a fraction $z$ of the jet transverse energy. Significant medium-modified softening of the JFF (by a factor of $\sim 4$ and slightly increasing with $z$ ) was obtained with PYQUEN/HYDJET simulations. In addition, the "anti-correlation" between softening of the JFF and suppression of the absolute jet rates due to partonic energy loss out of jet cone (wide-angle gluon bremsstrahlung and collisional loss) is predicted.

Jets induced by heavy quarks. The possibility to observe the medium effects in the channel with the production of B-jet tagged by an energetic muon was also analyzed. A significant softening of the b-jet fragmentation function (measured with the energetic muon) due to b-quark energy loss in QGP is predicted.

Azimuthal anisotropy of jet production. The azimuthal anisotropy of particle spectrum is characterized by the second coefficient of the Fourier expansion of particle azimuthal distribution, elliptic flow coefficient, $v_{2}$. The non-uniform dependence of medium-induced partonic energy loss in non-central heavy ion collisions on the parton azimuthal angle $\varphi$ (with respect to the reaction plane) is mapped onto the final jet spectra. The predicted by HYDJET/PYQUEN elliptic flow coefficients for jets $v_{2}^{\text {jet }}$ increases almost linearly with the growth of $b$ and becomes a maximum at $b \sim 1.6 R_{A}$ (where $R_{A}$ is the nucleus radius). After that, the $v_{2}^{\text {jet }}$ coefficients drop rapidly with increasing $b$. The maximum estimated value is $v_{2}^{\text {jet }} \sim 0.04$.

$P_{T}$-imbalance in dimuon tagged jet events. An important probe of medium-induced partonic energy loss in ultrarelativistic heavy ion collisions is production of a single jet opposite to a gauge boson such as $\gamma^{\star} / Z^{0}$ decaying into dileptons. The advantage of such processes is that the mean initial transverse momentum of the hard jet equal to the mean initial/final transverse momentum of boson, and the energy lost by the parton can be estimated from the observed $p_{T}$-imbalance between the leading particle in a jet and the lepton pair. Despite the fact that the initial distribution is smeared and asymmetric due to initial-state gluon radiation, hadronization effects, etc., the predicted by HYDJET/PYQUEN additional smearing and the displaced mean and maximum values of the $p_{\mathrm{T}^{-}}$ imbalance due to partonic energy loss can be significant. The $p_{\mathrm{T}}$-imbalance between the $\mu^{+} \mu^{-}$pair and a leading particle in a jet is directly related to the absolute value of partonic energy loss, and almost insensitive to the form of the angular spectrum of the emitted gluons and to the experimental jet energy resolution. 


\section{Summary}

Among other heavy ion event generators, HYDJET and HYDJET++ are concentrated on the detailed simulation of jet quenching effect based on the partonic energy loss model PYQUEN, and also reproducing the main features of nuclear collective dynamics by fast (but realistic) way. The final hadron state in HYDJET/HYDJET++ represents the superposition of two independent components: hard multi-parton fragmentation and soft hydro-type part. The hard parts of HYDJET and HYDJET++ are identical. The soft part of HYDJET++ contains the important additional features as compared with HYDJET (detailed treatment of thermal and chemical freeze-out hypersurfaces, resonance decays, etc.) HYDJET describes well the main high- $p_{T}$ observables in heavy ion collisions at RHIC, while HYDJET++ is capable of reproducing also the bulk event properties (such as hadron spectra and ratios, radial and elliptic flow, femtoscopic momentum correlations). Both generators are promising tools for the various simulation studies in heavy ion collisions at the LHC.

\section{Acknowledgments}

I.L. wishes to express the gratitude to the organizers of the Workshop "High-pT Physics at LHC" for the warm welcome and the hospitality. This work was supported by Russian Foundation for Basic Research (grants No 08-02-91001 and No 08-02-92496) and Grants of President of Russian Federation (No 1007.2008.2 and No 1456.2008.2).

\section{References}

[1] F. Carminati et al. (ALICE Collaboration), J. Phys. G 30 (2004) 1517.

[2] B. Alessandro et al. (ALICE Collaboration), J. Phys. G 32 (2006) 1295.

[3] D. d'Enterria et al. (CMS Collaboration), J. Phys. G 34 (2007) 2307.

[4] N. Armesto (ed.) et al., J. Phys. G 35 (2008) 054001.

[5] http://cern.ch/lokhtin/pyquen .

[6] T. Sjostrand, S. Mrenna and P. Skands, JHEP 0605 (2006) 026.

[7] I.P. Lokhtin and A.M. Snigirev, Eur. Phys. J. C 45 (2006) 211.

[8] R. Baier, Yu. L. Dokshitzer, A.H. Mueller and D. Schiff, Phys. Rev. C 60 (1999) 064902.

[9] R. Baier, Yu. L. Dokshitzer, A.H. Mueller and D. Schiff, Phys. Rev. C 64 (2001) 057902.

[10] http://cern.ch/lokhtin/hydro/hydjet.html .

[11] I.P. Lokhtin, L.V. Malinina, S.V. Petrushanko, A.M. Snigirev, I. Arsene, K. Tywoniuk, arXiv:0809.2708

[12] http://cern.ch/lokhtin/hydjet++ .

[13] N.S. Amelin et al., Phys. Rev. C 74 (2006) 064901.

[14] N.S. Amelin et al., Phys. Rev. C 77 (2008) 014903.

[15] R. Brun and F. Rademakers, Nucl. Instrum. Meth. A 389 (1997) 81; (http://root.cern.ch).

[16] K. Tywoniuk, I.C. Arsene, L. Bravina, A.B. Kaidalov and E. Zabrodin, Phys. Lett. B 657 (2007) 170.

[17] G. Torrieri et al., Comput. Phys. Coommun. 167 (2005) 229. 